\title{
A RATIONALIZATION OF UNUSUALLY LATE TRANSITION STRUCTURES FOR DICATION FRAGMENTATIONS
}

\author{
Peter M.W. GILL and Leo RADOM \\ Research School of Chemistry, Australian National University, Canberra, A.C.T. 2601, Australia
}

Received 4 February 1987

\begin{abstract}
$\mathrm{Ab}$ initio molecular orbital calculations indicate that the distance between the fragments in the transition structures for dissociation of doubly charged cations can be surprisingly large. We suggest a simple explanation for this behaviour and believe that it can be applied generally to such problems. In this way we have rationalized the dissociation characteristics of a number of representative dications.
\end{abstract}

\section{Introduction}

There has been extensive experimental and theoretical interest in the chemistry of gas-phase dications [1]. The fragmentation of such species into two monocations is often highly exothermic but may be inhibited by a significant barrier associated with the weakening of a covalent bond. The transition structure for fragmentation corresponds to the point at which the rate of increase in energy due to decreased covalent binding is equal to the rate of decrease in energy due to decreased Coulombic repulsion. In the case of a symmetric fragmentation,

$\mathrm{A}_{2}^{2+} \rightarrow \mathrm{A}^{+}+\mathrm{A}^{+}$,

the internuclear separation in the transition structure is typically $\approx 50 \%$ greater than in the equilibrium structure. For example, for the fragmentation of the recently observed [2] dihelium dication,

$\mathrm{He}_{2}^{2+} \rightarrow \mathrm{He}^{+}+\mathrm{He}^{+}$,

the He...He separation is $0.70 \AA$ in the equilibrium structure and $1.15 \AA$ in the transition structure [3].

We have noticed, on the other hand, that for asymmetric fragmentations of dications,

$\mathrm{AB}^{2+} \rightarrow \mathrm{A}^{+}+\mathrm{B}^{+}$,

the internuclear separation in the transition structure is often two or three times that in the equilibrium structure and the barrier to fragmentation can be remarkably high [4-6]. For example, for the reaction

$\mathrm{NH}_{3} \mathrm{NH}_{3}^{2+} \rightarrow \mathrm{NH}_{3} \mathrm{NH}_{2}^{+}+\mathrm{H}^{+}$

the N...H separations in the equilibrium and transition structures are 1.02 and $3.11 \AA$ respectively and the barrier to fragmentation is $523 \mathrm{~kJ} \mathrm{~mol}^{-1}[4,5]$. This result may seem surprising in that it implies that the balance between the rates of change of covalent binding and Coulombic repulsion is not achieved until quite large internuclear separations, at which stage the covalent binding must surely be very small. We present in this paper a rationalization of this rather perplexing behaviour.

\section{Method and results}

Using a modified version [7] of the GAUSSIAN 82 system of programs [8], standard ab initio calculations were carried out for each of the species indicated in table 1 and for the transition structures involved in each of the fragmentations.

The structures of all species were optimized at the Hartree-Fock (HF) level with the 6-31G* [9] basis set and the optimized geometries were confirmed as being equilibrium or transition structures by vibrational analysis. The spin-unrestricted formalism (UHF) was used for odd-electron species and for transition structures linking even- and odd-electron 
species. Although UHF theory is likely to underestimate the transition structure bond length of a homolytically breaking bond [10], it adequately describes the gross effects considered in this paper.

Full details of these calculations will be reported elsewhere [5].

\section{Results and discussion}

Table 1 and fig. 1 contain data pertaining to the dissociations of a variety of dications into charged fragments. The first three fragmentations exemplify symmetric bond cleavage reactions, typical of dimeric dications, in which the breaking bond lengthens by approximately $50 \%$ before the transition structure is reached. We quantify the dissociation behaviour by means of the bond-length ratio, $r_{\mathrm{Ts}} / r_{\text {eq }}$, which is roughly 1.5 in these cases. The five remaining examples are all asymmetric cleavages, in which the dications dissociate into two unequal parts. Of these examples, the first two possess bond-length ratios comparable to those found for the symmetric cases. However, for the last three, we find some extraordinarily late transition structures. Specifically, in the loss of a proton from either $\mathrm{N}_{2} \mathrm{H}_{6}^{2+}$ or $\mathrm{P}_{2} \mathrm{H}_{6}^{2+}$, the bond stretches to three times its equilibrium value as it approaches the transition structure and for the even more extraordinary case of the $\mathrm{MgH}^{2+}$ ion, the bond must distort to almost six times its equilibrium length before the transition structure is reached (at a bond distance of more than $12 \AA)$ !

What is the characteristic feature of the dications

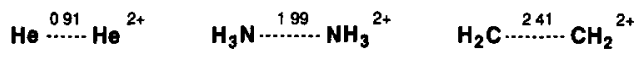

$$
\begin{aligned}
& \mathrm{H}_{3} \mathrm{~N} \cdots \cdots \cdots \mathrm{OH}_{2}{ }^{2+} \quad \mathrm{Cl} \cdots \cdots \cdots \cdot \mathrm{H}^{2+} \\
& \mathrm{H}_{3} \mathrm{NNH}_{2} \cdots \cdots \cdot \mathrm{H}^{2+} \quad \mathrm{H}_{3} \mathrm{PPH}_{2} \cdots \cdots \cdot \mathrm{H}^{2+} \quad \mathrm{Mg} \cdots 1245 \ldots \mathrm{H}^{2+}
\end{aligned}
$$

Fig. 1. Calculated (UHF/6-31G*) transition structure bond lengths.

whose dissociations involve such unusually stretched bonds in their transition structures?

First we consider the symmetric fragmentations. Initially, as the central bond is stretched beyond its equilibrium value, the loss in covalent binding energy (which decays exponentially) is greater than the gain afforded by the loss in Coulombic energy (an $r^{-1}$ effect). It is this that leads to an initial increase in energy accompanying stretching from the equilibrium structure. Soon, however, the diminished covalent energy is overwhelmed by the Coulombic repulsion, and the highly exothermic and irreversible fragmentation process becomes inevitable. The transition structure for this process corresponds to the point at which the rates of change of covalent binding and Coulombic repulsion are balanced. In the case of symmetric fragmentations, this is found to occur when the lengths of the central bonds in the transition structures for these cleavages are approximately 1.5 times their equilibrium values, for it is at such extensions that the covalent binding energy is sufficiently reduced.

Table 1

\begin{tabular}{|c|c|c|c|c|c|c|}
\hline $\mathrm{AB}^{2+} \rightarrow \mathrm{A}^{+}+\mathrm{B}^{+}$ & $r_{\text {eq }}$ & $r_{\mathrm{TS}}$ & $r_{\mathrm{Ts}} / r_{\mathrm{eq}}$ & $\operatorname{IE}_{\mathbf{a}}\left(\mathrm{A}^{+}\right)$ & $\mathrm{IE}_{\mathrm{a}}(\mathrm{B})$ & $\Delta$ \\
\hline $\mathrm{He}-\mathrm{He}^{2+} \rightarrow \mathrm{He}^{+}+\mathrm{He}^{+}$ & 0.66 & 0.91 & 1.4 & 5234 & 2262 & 2972 \\
\hline $\mathrm{H}_{3} \mathrm{~N}-\mathrm{NH}_{3}^{2+} \rightarrow \mathrm{H}_{3} \mathrm{~N}^{+}+\mathrm{NH}_{3}^{+}$ & 1.44 & 1.99 & 1.4 & 2193 & 817 & 1376 \\
\hline $\mathrm{H}_{2} \mathrm{C}-\mathrm{CH}_{2}^{2+} \rightarrow \mathrm{H}_{2} \mathrm{C}^{+}+\mathrm{CH}_{z}^{+}$ & 1.43 & 2.41 & 1.7 & 2011 & 804 & 1207 \\
\hline $\mathrm{H}_{3} \mathrm{~N}-\mathrm{OH}_{2}^{2+} \rightarrow \mathrm{H}_{3} \mathrm{~N}^{+}+\mathrm{OH}_{2}^{+}$ & 1.41 & 1.81 & 1.3 & 2193 & 1038 & 1155 \\
\hline $\mathrm{ClH}^{2+} \rightarrow \mathrm{Cl}^{+}+\mathrm{H}^{+}$ & 1.40 & 2.05 & 1.5 & 2170 & 1308 & 862 \\
\hline $\mathrm{H}_{3} \mathrm{NNH}_{2}-\mathrm{H}^{2+} \rightarrow \mathrm{H}_{3} \mathrm{NNH}_{2}^{+}+\mathrm{H}^{+}$ & 1.03 & 3.11 & 3.0 & 1490 & 1308 & 182 \\
\hline $\mathrm{H}_{3} \mathrm{PPH}_{2}-\mathrm{H}^{2+} \rightarrow \mathrm{H}_{3} \mathrm{PPH}_{2}^{+}+\mathrm{H}^{+}$ & 1.39 & 4.01 & 2.9 & 1368 & 1308 & 60 \\
\hline $\mathrm{MgH}^{2+} \rightarrow \mathrm{Mg}^{+}+\mathbf{H}^{+}$ & 2.22 & 12.45 & 5.6 & 1420 & 1308 & 112 \\
\hline
\end{tabular}

Bond lengths $(\AA)$ and ionization energies $\left(\mathrm{kJ} \mathrm{mol}^{-1}\right)$ in dissociating dication systems $\left(\mathrm{HF} / 6-31 \mathrm{G}^{*}\right)$ 


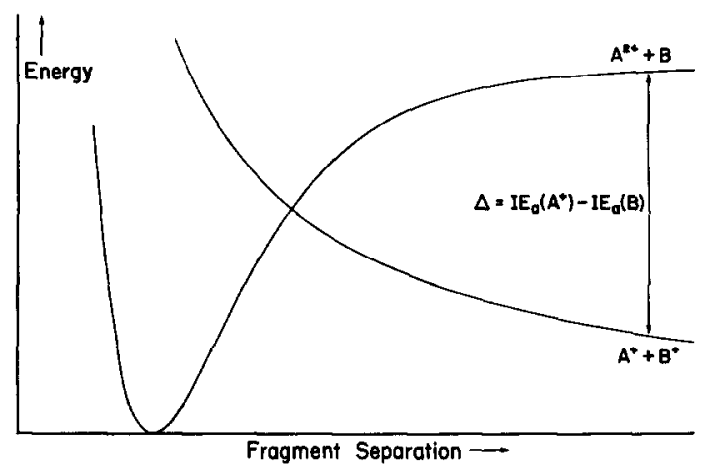

Fig. 2. Schematic representation of the zeroth-order potential curves for a general $A B^{21}$ system. It is the avoided crossing between these curves which produces kinetic stability in many thermodynamically unstable dications.

In the cases of some of the asymmetric fragmentations, however, things appear to be rather different. Apparently the above argument needs modification, since we would expect the covalent binding energy of a breaking bond almost entirely to have vanished when the transition structure bond lengths for the last three systems in table 1 are reached.

The key to understanding the data in table 1 is a more fundamental understanding of the origin of the shape of the potential curve describing the fragmentation of a dication.

The potential energy curve along the reaction coordinate for a dissociating $\mathrm{AB}^{2+}$ dication can usefully be considered as arising from an avoided crossing between a repulsive state which correlates with $\mathrm{A}^{+}+\mathrm{B}^{+}$and an attractive state which correlates with $\mathrm{A}^{2+}+\mathrm{B}$ ( fig. 2). (Throughout this paper we label the fragments so that the second ionization energy of $A$ is less than that of B.) Strictly, we should also include the attractive but higher-lying $A+B^{2+}$ curve (if it exists), but when the second ionization energies of $A$ and $B$ are sufficiently different its effect on the resulting ground-state potential curve will almost always be small.

Normally (at infinite separation) the $\mathrm{A}^{2+}+\mathrm{B}$ system will be higher in energy than the $\mathrm{A}^{+}+\mathrm{B}^{+}$system because it usually requires more energy to ionize a cation than a neutral. We can express this by defining

$\Delta=\mathrm{IE}_{\mathrm{a}}\left(\mathrm{A}^{+}\right)-\mathrm{IE}_{\mathrm{a}}(\mathrm{B})$

and noting that, as in fig. $2, \Delta$ will normally be posi-

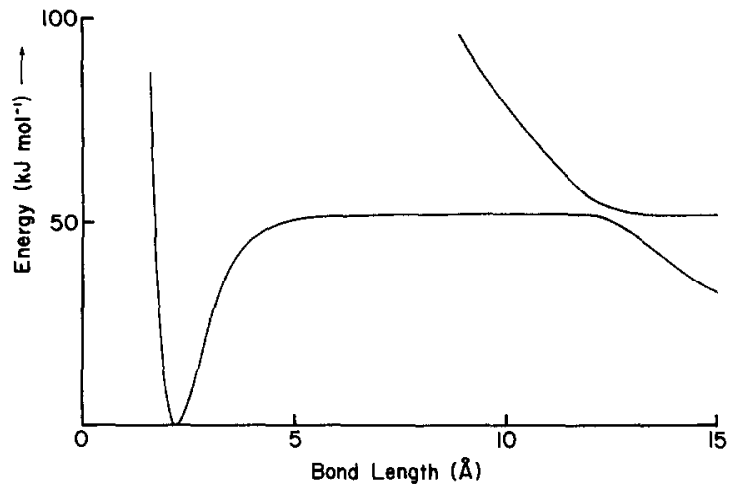

Fig. 3. Calculated (UHF/6-31G*) potential energy curves describing the dissociation of $\mathrm{MgH}^{2+}$. The extraordinarily late $[\mathrm{Mg}-\mathrm{H}]^{2+}$ transition structure arises from an avoided crossing at a very long bond length.

tive. The last three columns of table 1 reveal that this is true for all eight of the systems studied. Furthermore, upon comparing the $r_{\mathrm{TS}} / r_{\mathrm{eq}}$ and $\Delta$ columns of table 1 , and considering the effect of varying $\Delta$ in fig. 1 , we can now begin to understand the origin of the bond length ratios obtained.

In cases where $\Delta$ is large, the two curves will cross at a comparatively small fragment separation. Although we do not often know a priori the detailed behaviour of the resulting ground-state potential curve, we would expect it to exhibit a maximum (corresponding to the transition structure for the fragmentation) at a fragment separation close to that of the crossing point. On this basis, we predict that large $\Delta$ values will generally lead to early transition structures. Conversely, we predict that small $\Delta$ values will be associated with late transition structures. Moreover, it follows that we can construct a dication $\mathrm{AB}^{2+}$ (with appropriate $\Delta$ value) with almost any nominated bond-length ratio. In fact, the last of the eight examples in table 1 , the $\mathrm{MgH}^{2+}$ ion, was diliberately chosen in this way to display an extraordinarily long bond distance in its fragmentation transition structure (fig. 3 ).

It is also possible to interpret fig. 2 by means of a classical argument which serves to show the relationship between the avoided crossing model and the model for symmetric dissociations described earlier. In the equilibrium structure of a heteronuclear $\mathrm{AB}^{2+}$ system, the charge will be centred largely on the more easily ionized moiety, that is, on A. For example, in 
$\mathrm{MgH}^{2+}$ at its equilibrium structure, the magnesium bears a charge of $\delta=+1.68$ (UHF/6-31 G*, Löwdin analysis) and the $\mathrm{H}$ is therefore better described as a hydrogen atom than as a proton. One implication of this uneven distribution of charge is that the repulsive Coulomb energy between the incipient fragments, which is given

$$
\begin{aligned}
E & =q_{\mathrm{A}} q_{\mathrm{B}} / r \\
& =1.68 \times 0.32 / r=0.54 / r \quad\left(\text { for } \mathrm{MgH}^{2+}\right)
\end{aligned}
$$

is only a little more than half of that which would arise if the $\mathrm{Mg}$ and $\mathrm{H}$ shared the charge evenly. However, as the molecule is stretched toward the transition structure, the positive charge on $B$ must rise (since eventually it will be exactly unity) and consequently, as the denominator increases, the numerator in (6) will also increase. It follows therefore that, as the transition structure is approached, the Coulomb repulsion should not be expected to decrease hyperbolically as in the symmetric dissociations. It is even possible in principle for the Coulomb repulsion to increase initially. The consequence of these considerations is that the point at which the rate of decay of Coulomb repulsion matches the rate of decay of covalent binding (the transition structure) may occur much later than might initially have been anticipated.

The arguments presented in this article bear some relevance to the proposal of Beynon and co-workers [11] that the experimentally measured kinetic energy release in the dissociation of a dication may be equated to the residual repulsive Coulombic potential energy at the transition structure. Implicit in this approximation is the assumption that the charges on both of the fragments in the transition structure are close to unity. In fig. 2 we find support for this assumption. Although in the equilibrium structure, fragment $A$ is likely to bear more of the positive charge than $B$, the transition structure occurs at the point where the system passes onto the $\mathrm{A}^{+}+\mathrm{B}^{+}$ curve, consistent with the Beynon proposal.

The question also arises as to how a system with a negative $\Delta$ value (for example, $\mathrm{CaH}^{2+}$ ) would dissociate. It is clear from the present argument that such a molecule will dissociate, without reverse activation energy, to form $\mathrm{A}^{2+}+\mathrm{B}$. The channel leading to $\mathrm{A}^{+}+\mathrm{B}^{+}$is energetically unfavourable, both kinetically and thermodynamically.

It is possible, for a sufficiently late transition struc- ture, i.e. when $\Delta$ is small, to derive a simple approximate formula for the transition structure bond length in a diatomic dication, even if nothing is known of the chemical interactions in the $[\mathrm{A}-\mathrm{B}]^{2+}$ system. We begin by noting that the $\mathrm{A}^{2+}+\mathrm{B}$ curve approaches its limiting energy value much faster than does the $\mathrm{A}^{+}+\mathrm{B}^{+}$curve (fig. 2) and, at large fragment separations, may be considered constant. With the additional assumption that the $\mathrm{A}^{+}+\mathrm{B}^{+}$curve is hyperbolic (as would be anticipated from Coulomb's law), it follows that the crossing point, and therefore the estimated transition structure bond length, is given by

$$
\begin{aligned}
r_{\mathrm{TS}} & =\Delta^{-1} \quad\left(\Delta \text { and } r_{\mathrm{Ts}} \text { in au }\right) \\
& =1389 \Delta^{-1}\left(\Delta \text { in } \mathrm{kJ} \mathrm{mol}^{-1}, r_{\mathrm{TS}} \text { in } \AA\right) .
\end{aligned}
$$

For the case of $\mathrm{MgH}^{2+}$, eq. (8) predicts a transition structure bond length of $12.40 \AA$, in close agreement with the directly calculated value of $12.45 \AA$, demonstrating that the assumptions leading to formulae (7) and (8) are valid when $\Delta$ is sufficiently small.

This formula clearly has useful predictive value. Suppose, for example, that we were interested in the heterolytic dissociations of the $\mathrm{HX}^{2+}$ dications, where $X$ is a halogen. Having discovered that the second ionization energies of the halogens $\mathrm{F}, \mathrm{Cl}, \mathrm{Br}$ and I decrease monotonically from 3381 to $1852 \mathrm{~kJ}$ $\mathrm{mol}^{-1}$, and knowing that the ionization energy of $\mathrm{H}$ is $1318 \mathrm{~kJ} \mathrm{~mol}^{-1}$ [ 12 ], we can estimate that the transition structure bond lengths will increase monotonically from less than $1 \AA$ (for $\mathrm{HF}^{2+}$, implying little or no barrier to dissociation) to nearly $3 \AA$ (for $\mathrm{HI}^{2+}$ ).

\section{Conclusion}

In this Letter we have proposed a simple model to explain the surprisingly wide variation in transition structure bond lengths in dissociating dications. In the case of late transition structures, our model allows a semi-quantitative prediction of the length of the breaking bond in the transition structure, based only on a knowledge of the ionization energies of the fragments formed.

\section{Acknowledgement}

We thank Professor J.A. Pople for very helpful discussions regarding this manuscript. 


\section{References}

[1] W. Koch, F. Maquin, D. Stahl and H. Schwarz, Chimia 39 (1985) 376;

W. Koch and H. Schwarz, in: Structure/reactivity and thermochemistry of ions, ed. P. Ausloos, NATO ASI series, to be published, and references therein.

[2] M. Guilhaus, A.G. Brenton, J.H. Beynon, M. Rabrenovic and P. Von R. Schleyer, J. Phys. B17 (184) L605.

[3] H. Yagisawa, II. Sato and T. Watanabe, Phys. Rev. A16 (1977) 1352.

[4] W.J. Bouma and L. Radom, J. Am. Chem. Soc. 107 (1985) 345.

[5] P.M.W. Gill and L. Radom, to be published.

[6] M.W. Wong, R.H. Nobes and L. Radom, to be published.

[7] J. Baker, R.H. Nobes, D. Poppinger and M.W. Wong, unpublished;
J. Baker, J. Comput. Chem. 7 (1986) 385.

[8] J.S. Binkley, M.J. Frisch, D.J. DeFrees, K. Raghavachari, R.A. Whiteside, H.B. Schlegel, E.M. Fluder and J.A. Pople, GAUSSIAN 82, Carnegie-Mellon University, Pittsburgh.

[9] W.J. Hehre, R. Ditchfield and J.A. Pople, J. Chem. Phys. 56 (1972) 2257;

P.C. Hariharan and J.A. Pople, Theoret. Chim. Acta 28 (1973) 213.

[10] P.M.W. Gill and L. Radom, Chem. Phys. Letters 132 (1986) 16.

[11] J.H. Beynon and A.E. Fontaine, Chem. Commun. (1966) 717 ;

R.G. Cooks, J.H. Beynon, R.M. Caprioli and G.R. Lester, Metastable ions (Plenum Press, New York, 1978).

[12] G.H. Aylward and T.J.V. Findlay, SI chemical data (Jacaranda Wiley, Milton, 1974). 